\title{
Joint dependent passive stiffness in paretic and contralateral limbs of spastic patients with hemiparetic stroke
}

\author{
J D Given, J P A Dewald, W Z Rymer
}

\begin{abstract}
Torque-angle relations at the elbow and ankle joints of relaxed normal controls and patients with hemiparetic stroke were compared. Low velocity flexion/ hold/extension angular perturbations were applied to the joint under examination. The resulting torque-angle profiles described a hysteresis loop with similar slopes during the extension and flexion stages but separated by a vertical torque offset. Torque-angle responses obtained in the absence of significant muscle activation, as recorded by surface electromyographic activity, were designated as passive.

Elbow passive stiffness estimates were calculated from the slope of the torqueangle response during the flexion stage of the perturbation. The elbow torque-angle plots exhibited linear passive stifiness with magnitude significantly lower than the passive stiffiness of the ankle in both normal subjects and spastic patients. Changing ramp velocity had no significant effect on the passive torque-angle hysteresis loop at the elbow.
\end{abstract}

A comparison of the torque-angle relations between hemiparetic spastic and normal control arms showed no significant differences in passive stiffness. Furthermore, no significant differences were found between paretic and contralateral upper limbs of a given hemiparetic subject. By contrast, significant differences in the torque-angle hysteresis loop were present between the paretic and contralateral ankles in all hemiparetic patients tested. These differences were more significant during dorsiflexion, and therefore seem to be related to preferential changes in mechanical properties of plantar flexor muscles.

It is hypothesised that the differences in the torque-angle hysteresis loop between elbow and ankle joints are related primarily to the larger amount of connective tissue in the calf muscles, as well as to a larger total physiological cross sectional area of calf muscles compared with elbow muscles.

It is further hypothesised that the preferential increases in passive stifiness at the ankle in spastic legs result from immobilisation induced changes in muscle connective tissue, which are most prominent in muscles with predominantly slow-twitch fibres (such as soleus).
Connective tissue surrounding such slow twitch muscle fibres have been shown to be more sensitive to immobilisation than those in fast twitch muscle. The functional, pathophysiological, and clinical implications of our findings are reviewed.

(F Neurol Neurosurg Psychiatry 1995;59:271-279)

Keywords: Stroke; passive stiffness; spasticity

Increases in stiffness of the spastic limb are generally attributed to spasticity, which is, in turn, related to abnormal stretch reflex behaviour. ${ }^{1}$ The increased resistance perceived by the clinician could, however, also be produced by changes in the underlying mechanical properties of muscle and tendon. ${ }^{23}$ The total mechanical resistance to an externally imposed change in joint angle is the result of the combined contributions of passive tissues and active contractile properties of the muscle-tendon complex, so that the presence of increased stiffness cannot be automatically attributed to an enhanced stretch reflex. ${ }^{4-7}$ It follows that accurate quantification of spasticity requires that passive mechanical properties of muscle and tendon be estimated first. Passive stiffness properties can be derived from measured torque-angle responses obtained in the absence of significant muscle activation (as judged from electromyographic (EMG) recordings). The present study was designed to characterise and compare passive stiffness of the elbow and ankle joints in normal subjects and spastic patients, using quantitative mechanical measures with concurrent EMG recordings. Portions of this work have appeared in abstract form. ${ }^{89}$

\section{Methods}

SUBJECTS

The subject pool consisted of 10 spastic patients with hemiparetic stroke and 10 normal volunteers. The selection criteria used for the hemiparetic spastic patients were; $(a)$ paresis confined to one side; $(b)$ presence of abnormal muscle tone; $(c)$ presence of normal passive range of motion in the impaired ankle or elbow; (d) absence of severe cognitive or affective dysfunction; $(e)$ absence of severe concurrent medical problems; $(f)$ capacity to provide informed consent.

Subjects were examined by a licensed physical therapist to verify their admissibility to the 
study. Muscle tone was evaluated with the modified Ashworth scale from 0-5, where 0 signifies normal tone and 5 indicates severe spasticity. ${ }^{10}$ The range of motion (ROM) was measured for elbow flexion and extension with a goniometer. Our hemiparetic experimental group of 10 patients (eight men, two women) ranged in age from 34 to 72 (mean 52(SD 11)) with Ashworth scores from 2 to 4 . All subjects provided informed consent in writing before participating in this study, which was reviewed and approved by the Institutional Review Board of Northwestern University.

\section{EXPERIMENTAL PROTOCOL}

A slow ramp position perturbation protocol was applied to the joint in question (ankle or elbow) to assess the passive mechanical properties of the joint.

In our studies of the elbow, the subject was tightly strapped into a specially designed chair with the shoulder abducted $85^{\circ}$ and held in a mid-flexion/extension position. The elbow rested in a shallow cup above the axis of rotation of the motor. The wrist, hand, and lower forearm were casted with the forearm held in a mid-prosupinated position, and the cast was then attached with a $U$ bolt to a lightweight beam coupled to the motor. Angular position of the joint was measured by a potentiometer and velocity by a tachometer, each mounted on the motor shaft. Torque was measured by a torque transducer mounted between the motor shaft and the beam. Electromyographic activity (EMEG) in the biceps brachii, brachioradialis, and lateral triceps brachii muscles was recorded using Medtronic $\mathrm{Ag} / \mathrm{AgCl}$ surface electrodes (CLEARTRACE LT 1710-003).

The subject's arm was positioned at about $120^{\circ}$ of elbow extension $\left(180^{\circ}\right.$ is designated as full extension). The arm was moved through a flexion arc of one radian $\left(57.3^{\circ}\right)$ at a constant angular velocity, followed by a three to five second hold and then returned with the same stretch velocity to the initial starting position.

A similar protocol was used for the ankle. The subject lay in the lateral position with the knee and pelvis stabilised with restraining straps and firm bolsters. The foot was casted to a platform coupled to the motor. The centre of rotation of the ankle was lined up with the axis of rotation of the motor. EMGs were recorded over the anterior tibialis, soleus, and the medial and lateral gastrocnemius muscles. The foot was placed initially at about $30^{\circ}$ plantar flexion and then moved through an arc of $30^{\circ}$ in dorsiflexion at a constant velocity, followed by a hold phase. It was then returned with the same stretch velocity to the starting position. The knee was kept at a $30^{\circ}$ flexion angle.

For all trials, the subject was instructed to relax completely and not to intervene. The subject's relaxed state was verified by monitoring relevant EMGs. The angular range and velocity were varied for each subject to allow measurement of joint torque under passive conditions.

\section{DATA ANALYSIS}

A constant velocity protocol was used so that the measured torque response of the limb would be independent of limb inertia, once a steady state in velocity was achieved. Furthermore, if there is constant stiffness and viscosity over the angular range, a plot of torque $v$ position should produce a straight line response (this was usually the case at the elbow; see results). The slope of this line can then be calculated readily, and provides an estimate of joint stiffness $\mathrm{K}$. This stiffness is a measure of the amount of resistance to changes in position, and would contribute to the clinical perception of resistance at a joint.

For reliable identification and estimation of joint passive stiffness, stiffness measures must be repeatable and not vary with time over the course of the recordings. This invariance was confirmed by analysis of the stationarity of the experimental data. (Stationarity, which is a measure of time dependent variation in system response, was evaluated by comparing torque values at the midpoint and end of stretch across sequential trials). No significant trends were found. Estimation of passive stiffness measures was restricted to the constant velocity portion of the positional perturbation, where the torque-angle response was roughly linear (see previous paragraph). For the elbow joint, the average passive stiffness was estimated from the slope of the torque-angle curve covering the angular range from around $105-110^{\circ}$ to $65-70^{\circ}$. A least squares linear regression was used to estimate the slope (passive stiffness) of the torque-angle hysteresis loop during the flexion stage of the position perturbation protocol. Linear regression analysis was not done for the ankle joint as the torque-angle curves exhibited curvilinear behaviour and therefore a straight line approximation of passive stiffness over the constant velocity range of stretch was inappropriate.

Figure 1 shows a representative example of the elbow torque-angle responses of the affected limb in a relaxed hemiparetic patient, with corresponding EMG, for two different ramp velocities $(0.1 \mathrm{radians} / \mathrm{s}$ and $0.33 \mathrm{radi}-$ ans/s). A ramp stretch velocity of $0.33 \mathrm{radi}$ ans/s was sufficient to evoke a stretch reflex response in the affected upper limb, as indicated by torque and EMG responses in fig 1 . At the slower ramp stretch velocity, no stretch reflex was initiated, as can also been seen in fig 1. At these ramp stretch velocities, the effects of limb inertia were small and brief, and did not contaminate the measured torque response during the constant velocity region.

To eliminate trials with non-quiescent EMG activity from further analysis, we used a $95 \%$ confidence interval $(95 \% \mathrm{CI})$ derived from the resting baseline EMG measures. The identified quiescent trials were subsequently used to characterise the subject's passive torque-angle hysteresis loop. Ensemble mean values (mean value calculated across trials for each point in time) and associated $95 \%$ CIs were then calculated for both torque and rectified EMGs. The mean torque and EMG responses were plotted $v$ angular position for a given velocity. 
Figure 1 Torque and EMG measured from the relaxed impaired upper extremity of the most spastic patient at 6 and 20 degrees/s. Note the absence of stretch reflex response at the affected side at the lower velocity and a clear stretch reflex onset at the higher velocity which can be appreciated in both EMG and torque-angle profiles.

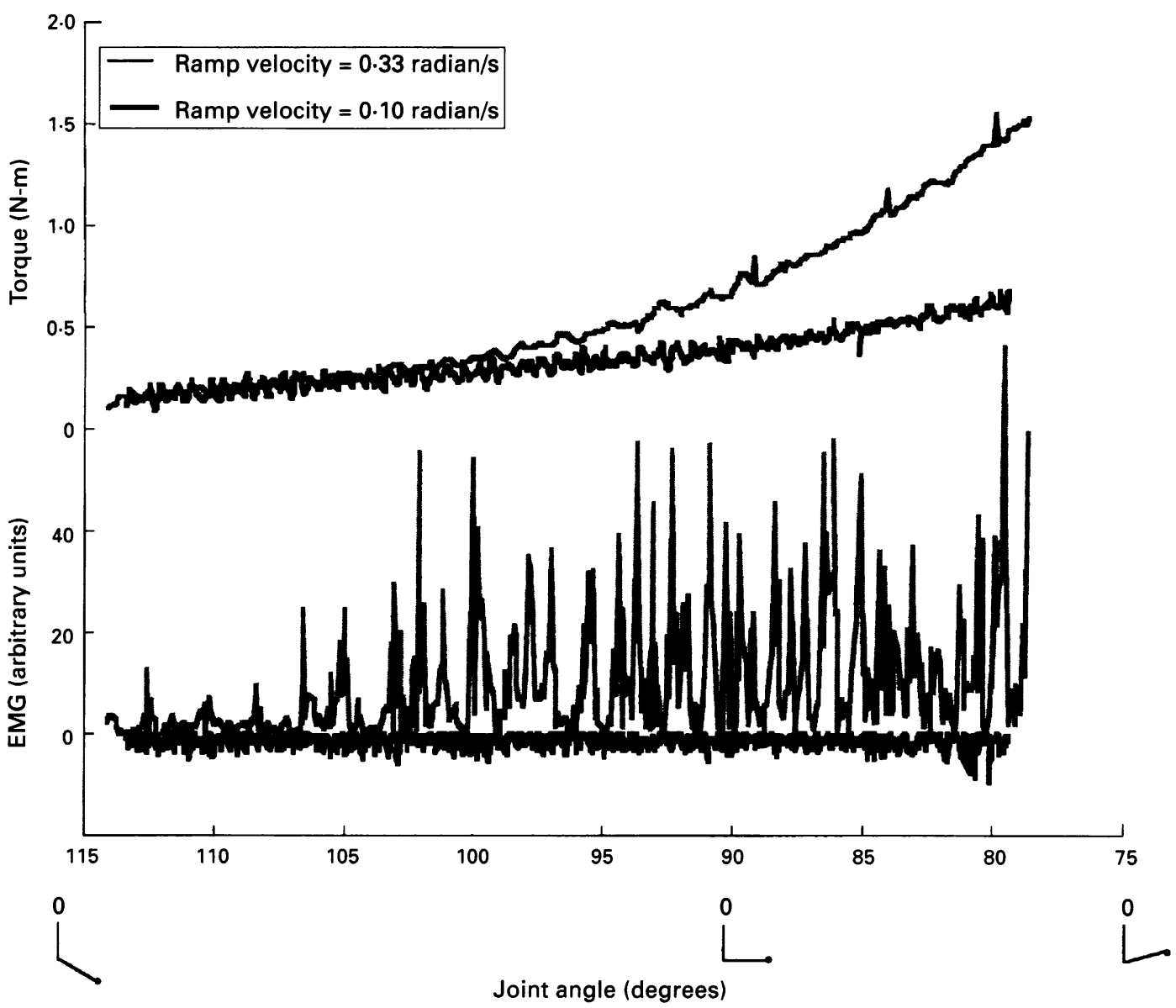

Mass of elbow muscles was estimated (indirectly) in our normal control group by measuring the maximum circumference of the relaxed upper arm between elbow and shoulder with the elbow at a $90^{\circ}$ flexion angle. We assume that the circumference, which is a measure of cross sectional area, is directly related to net muscle mass.

A non-parametric rank order correlation analysis, equivalent to Pearson's correlation coefficient, was used to measure the degree of association between limb circumference and the passive stiffness response. Spearman's rank correlation coefficient $(\rho)$ was chosen since the circumference measure provided rank ordering of muscle bulk between subjects. Ranking of the passive stiffness relations of the normal control group was then straightforward. An advantage of non-parametric rank order correlation analysis is that a linear relation need not be assumed.

\section{Results}

We compared torque-angle responses and stiffness estimates for spastic-paretic and contralateral limbs in patients with hemiparetic stroke and in the limbs of normal controls. The torque-angle response for each limb was consistent and showed very little trial to trial variability for subjects drawn from either the normal control or hemiparetic stroke groups (fig 2). Figure 2 (top) is the mean passive torque-angle response (in black), with the corresponding $95 \% \mathrm{CI}$ (shaded grey area) for the right elbow of a normal subject. Figure 2 (bottom) is the mean passive torque-angle response for the affected ankle of a patient.

TORQUE-ANGLE RELATIONS IN UPPER LIMBS OF SPASTIC PATIENTS AND NORMAL SUBJECTS

Torque-angle response data at the elbow were collected from a normal control group $(n=10)$ and the affected upper limb of 10 patients with hemiparetic stroke. The angular range between $110-105^{\circ}$ and $70-65^{\circ}$ was used to calculate passive stiffness estimates during the flexion stage. The non-linear behaviour of the torque response below $70^{\circ}$, seen in some hemiparetic patients (and also in normal controls) was due to the forearm physically coming into contact with the upper arm and subsequent compression of muscle mass. Although inter-trial variability for a subject's elbow was small, significant differences in elbow torqueangle responses were identified between subjects within each subpopulation (control and hemiparetic). As a consequence, the average normal response could not be described by a single torque-angle response but was better described by a range of normal response curves.

An intergroup comparison of torque-angle relations between control limbs (dominant or non-dominant, $n=10$ ) and impaired upper limbs of patients with hemiparetic stroke $(n=10)$ was performed to determine whether there was a higher mean joint passive stiffness within the stroke group. Figure 3 plots a representative group of torque-angle relations 


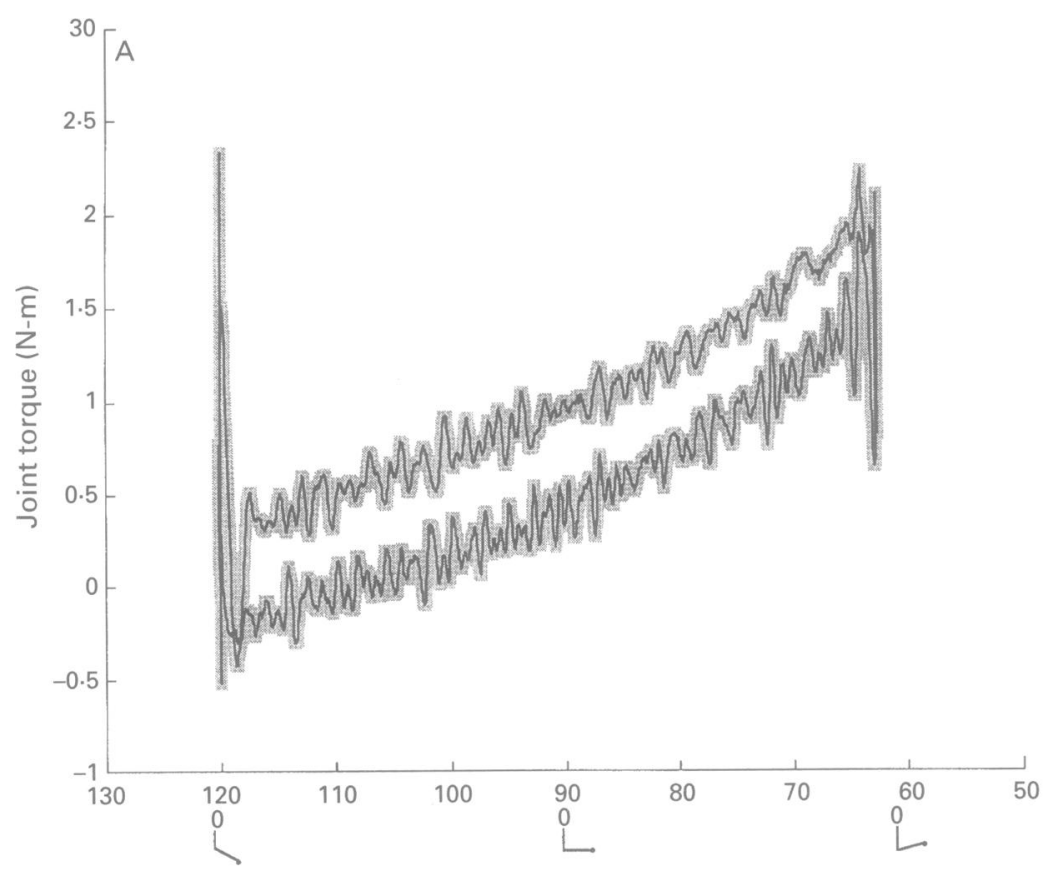

Joint angle (degrees)

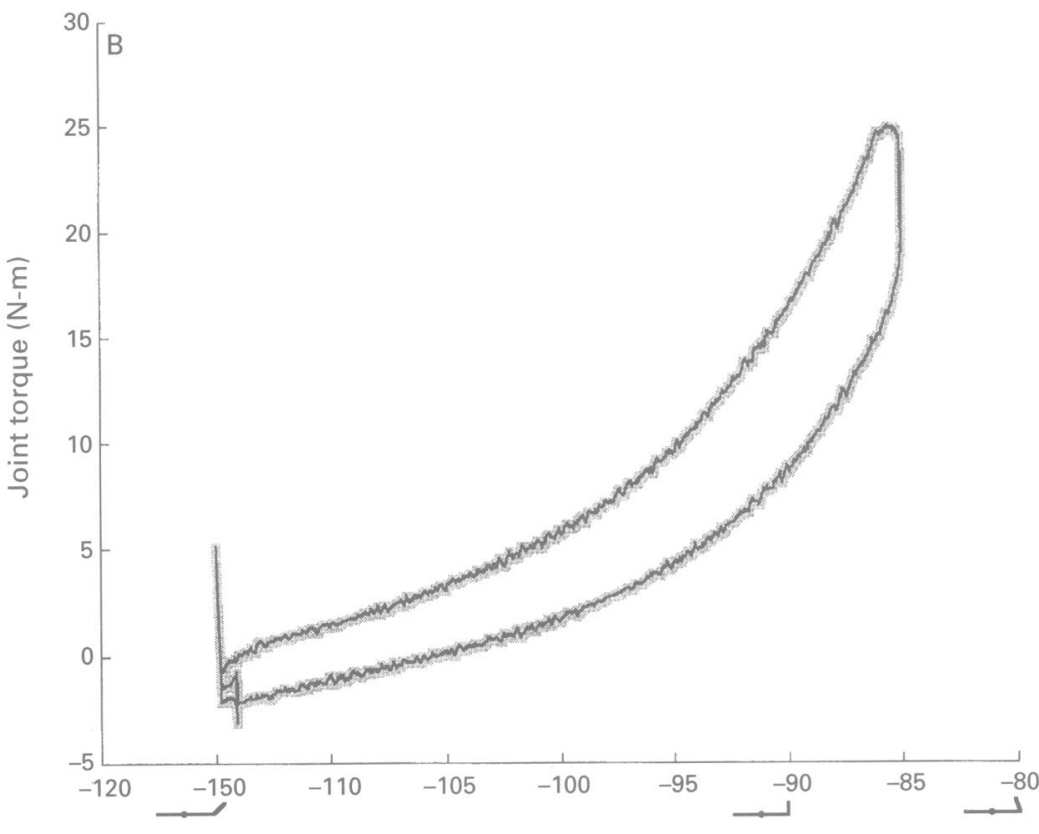

Joint angle (degrees)

Figure 2 Variability of torque-angle responses for the elbow of a normal control subject (top) and the ankle of a stroke patient (bottom). As can be appreciated by the narrow confidence bandwidth, the data provided reliable and consistent passive torque-angle responses for a given individual and ramp velocity. The angular range and least-squares estimation of the passive stiffness response for the stroke patient are shown.

from the affected elbows of the hemiparetic stroke population together with a shaded area which delineates the range of elbow passive stiffness responses in the normal control group. It is clear that the passive stiffness magnitudes in hemiparetic stroke fell well within the normal range. The passive joint stiffness of both test populations ranged from 0.008 to 0.036 Newton-meters $(\mathrm{N}-\mathrm{m}) /$ degree (fig 3).
LEFT AND RIGHT COMPARISON OF ELBOW TORQUE-ANGLE RESPONSES IN THE SAME SUBJECT

As described, passive stiffness estimates from the affected arms of the hemiparetic stroke population $(n=10)$ fell within the estimated normal range. Given the wide range of normal responses, however, significant within-subject (affected $v$ contralateral elbow) differences were still possible. Therefore, a within subject comparison of upper limbs was performed with a random subpopulation of five subjects from the normal control group and five of the 10 patients with hemiparetic stroke. The ensemble mean average torques of both arms were compared at each sampled angular position for the presence of significant differences in torque. A level of significance of 0.05 was used. The error bounds at each sampled point were $\pm 0.025-0.040 \mathrm{~N}-\mathrm{m}$, which corresponds to $1-3 \%$ of the peak to peak torque range. No statistically significant intrasubject differences were found between upper limbs of either population.

CORRELATION OF TORQUE-ANGLE RESPONSES WITH MUSCLE MASS IN THE UPPER EXTREMITY To explain the between subject variability of the elbow passive stiffness response, a correlation analysis, based on Spearman's correlation coefficient $\rho$ (see data analysis), was performed which compared upper limb muscle mass against the measured torque-angle response. We used limb circumference as a rough estimate of cross sectional limb diameter, and indirectly of muscle mass. This circumference was used to rank the subjects' muscle mass from smallest to greatest, whereas a similar ranking was derived for passive stiffness from values calculated from the passive torque-angle responses of the normal control group. A significant positive rank order Spearman correlation coefficient $(\rho=$ 0.82 , significant at the 0.01 level) was found between circumference and estimated elbow passive stiffness response in the normal control group. Figure 4 shows the mean torqueangle relations, ranked according to the passive stiffness estimates of three subjects. These subjects were chosen to reflect differences in limb circumference (and presumably in muscle mass). For this phase of the study, the number of normal control subjects was nine (one subject did not provide relaxed responses).

In summary, the estimated elbow passive stiffness responses of the hemiparetic stroke population were within normal limits. Furthermore, there was no significant intrasubject (left/right) variability for either the control or stroke populations and intertrial variability was small and consistent for both groups. This was not the case for the ankle, however, as will be discussed next.

LEFT AND RIGHT DIFFERENCES IN TORQUEANGLE RELATIONS AT THE ELBOW AND ANKLE JOINTS

To investigate potential differences in passive stiffness at different joints within the same 
Figure 3 Passive stiffness responses from five representative examples of the hemiparetic stroke population along with the population along with the passive stiffness profiles. Note that for the range of motion tested, all spastic upper limbs remain within the normal range.
Figure 4 Passive torqueangle responses as a function of muscle bulk for a subset of three normal control subjects. As the arm circumference increases so does the passive stiffness response.

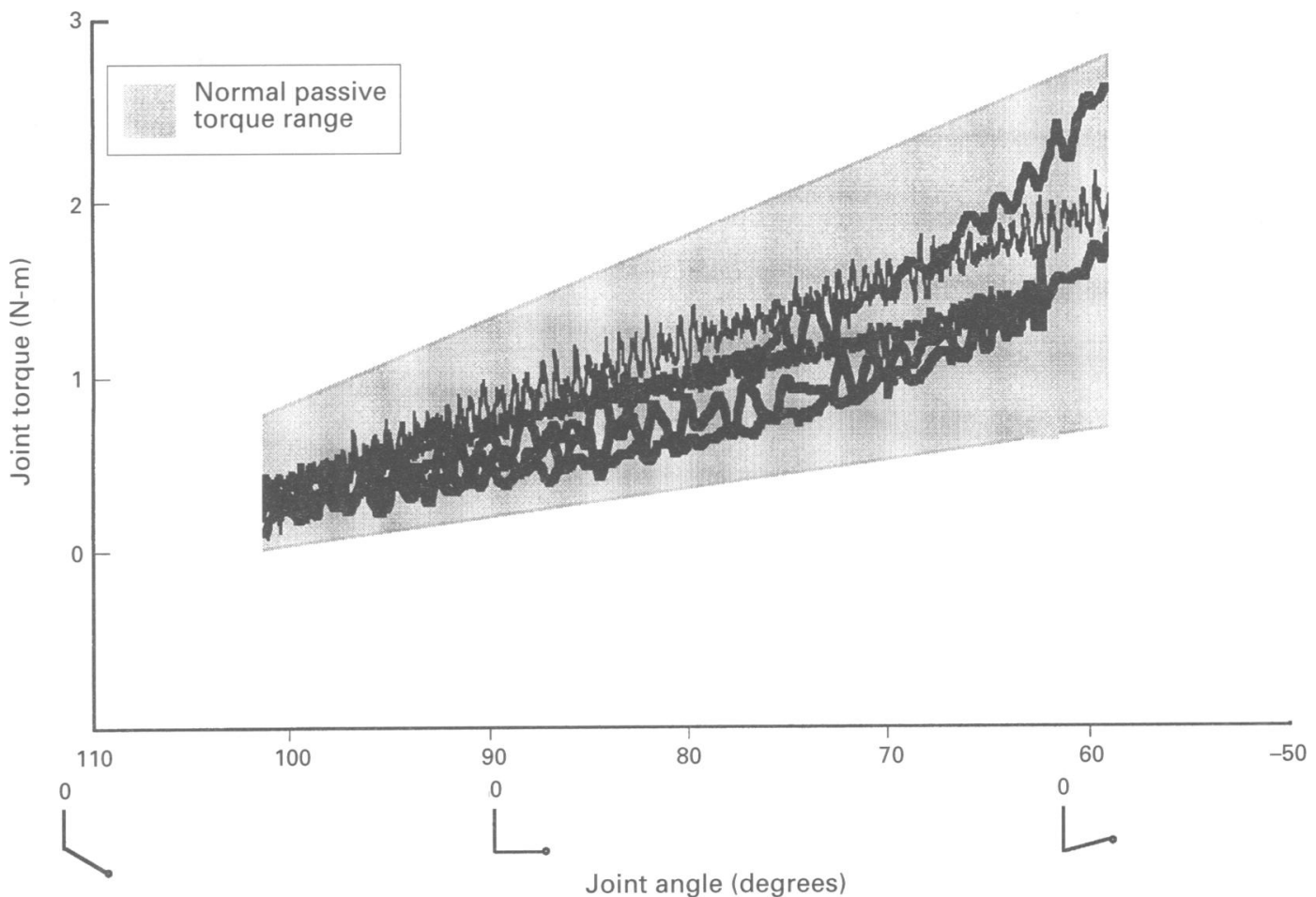

subject, we compared left/right torque-angle relations at the elbow and ankle joints of the same subject. The observed torque hysteresis loops displayed consistently greater slopes and vertical torque offsets at the ankle.

Furthermore, significant differences in passive stiffness were routinely seen between impaired and contralateral ankles of hemiparetic patients.

The passive torque-angle relation for a nor-

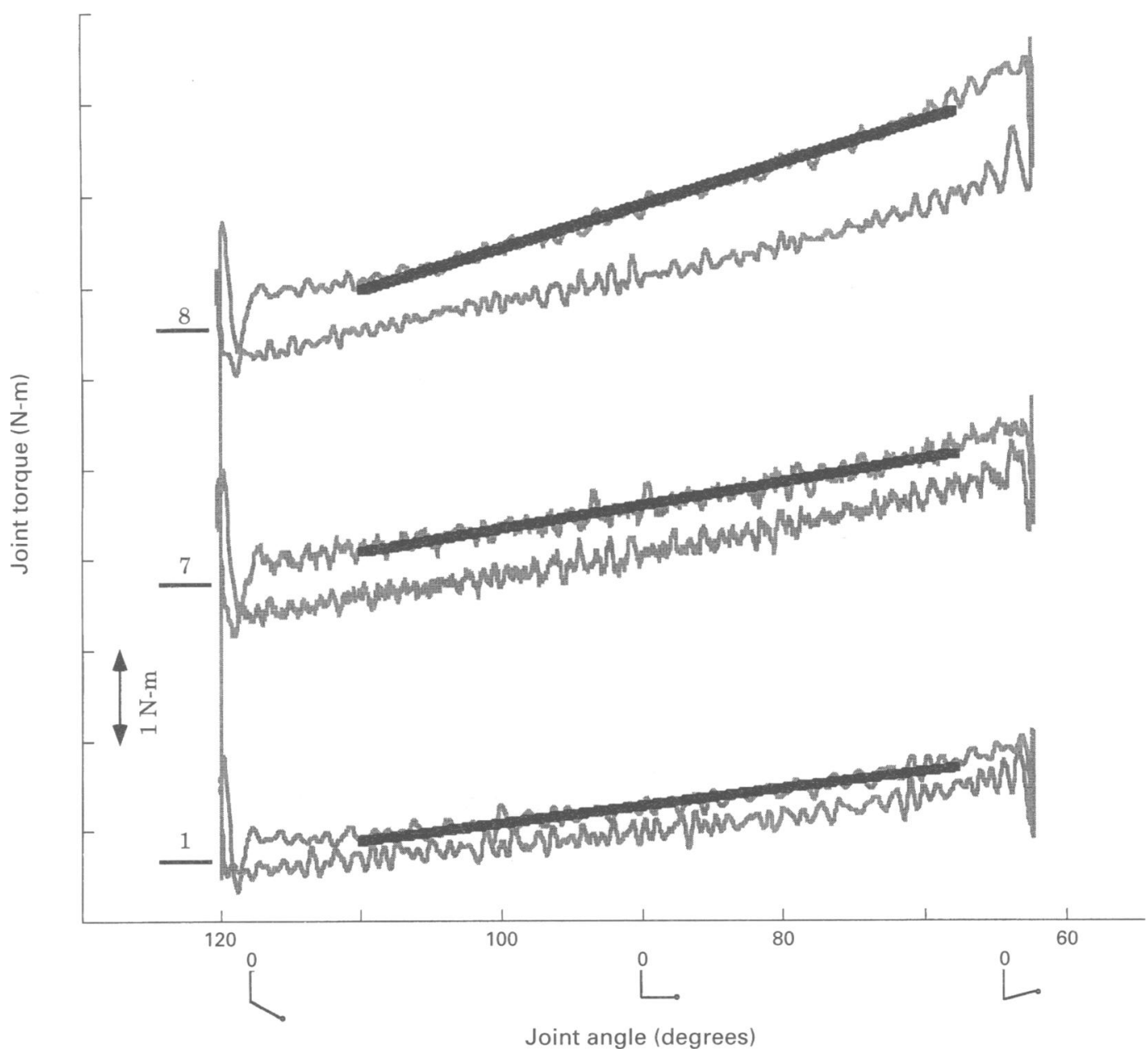



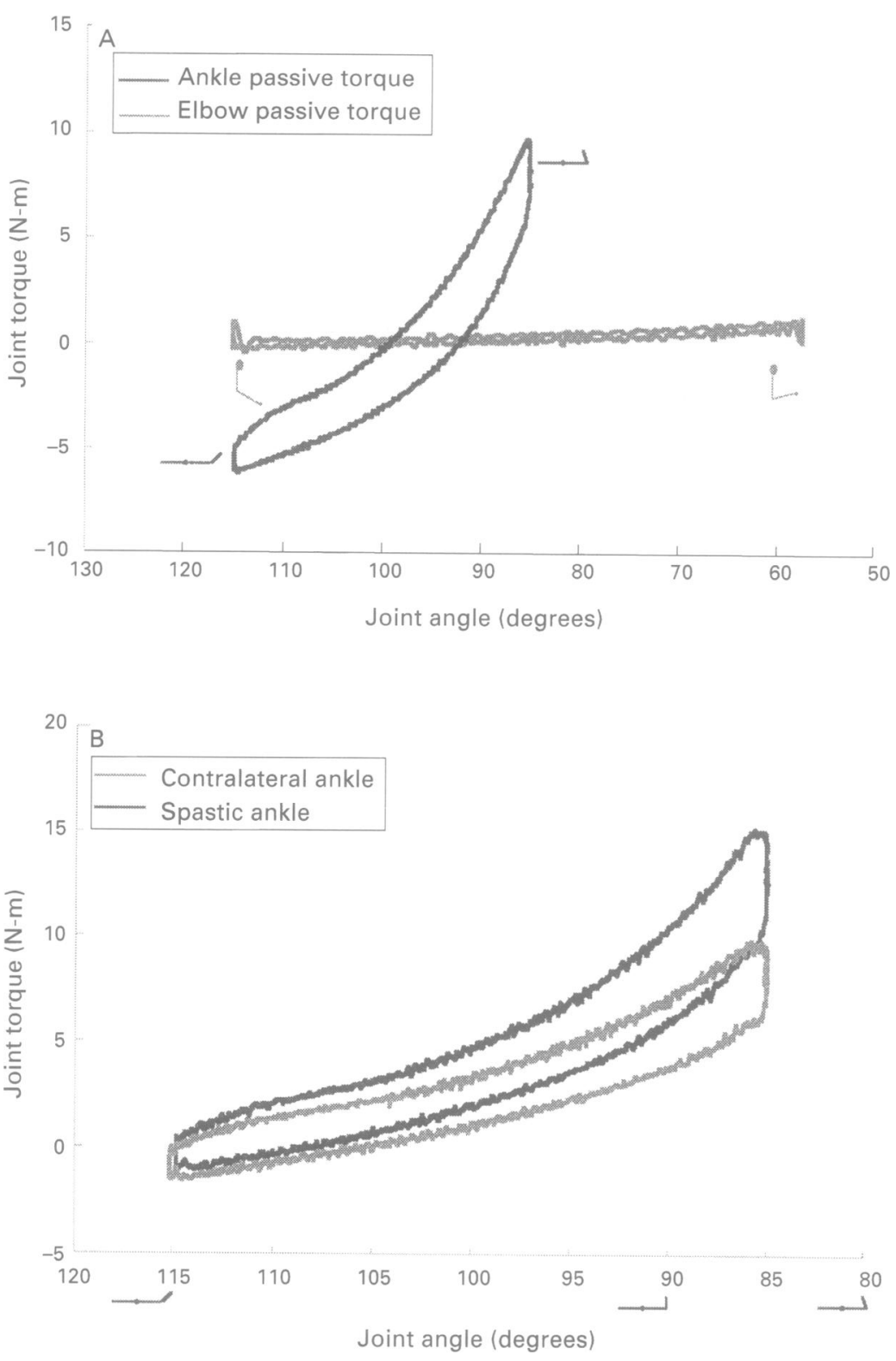

Figure 5 (A) Comparison of the passive torque-angle hysteresis loops of the ankle $v$ elbow for a normal control subject. The significantly higher passive stiffness at the ankle is striking. (B) Passive torque-angle hysteresis loops in the impaired v unimpaired ankle of a moderately spastic patient. Note the significant increases in passive stiffness at the spastic ankle relative to the contralateral side. mal control subject describes a hysteresis loop (fig 5A). The figure illustrates the rapid rise in torque during ankle dorsiflexion, as opposed to that at the elbow. Although the actual torque-angle responses differed substantially in the normal population, the absence of significant left and right variation was common to both joints.

Similar right-left comparisons of torqueangle relations were made at the ankle for a subset of the hemiparetic test population $(n=3)$. Significant differences in torque-angle relations were found between the impaired and contralateral ankles. Figure 5B shows an example of paired torque-angle profiles of the impaired and contralateral ankle joints for a hemiparetic patient. Differences between the impaired and contralateral ankle torqueangles profiles were quantified from peak torque values. For the dorsiflexed ankle position $\left(<90^{\circ}\right)$, significant increases in peak torque $(>40 \%)$ were found in the impaired limb (relative to the contralateral limb) for all three patients. In the plantar flexed position, peak torque differences were less than $4 \%$. These results indicate that there were preferential changes in the plantar flexors compared with the ankle dorsiflexors.

EFFECT OF MOTION VELOCITY ON TORQUEANGLE RELATIONS IN NORMAL AND HEMIPARETIC UPPER LIMBS

Changes in ramp velocity did not have a significant effect on passive torque-angle relations recorded at the elbow, at least over the range of velocities used (0.1-1.5 radians/s). This conclusion is based on data analysis with a subset of normal controls and hemiparetic patients ( $n=4, n=3$ respectively). The passive torque-angle relation described a hysteresis loop, which exhibited constant peak to peak torques and constant vertical offsets, independent of ramp stretch velocity (fig 6). Passive torque-angle responses, compared at each sampled angular position, were not significantly different (at the 0.05 level) across different velocities.
Figure 6 Elbow mean passive torque-angle response (in black) and corresponding $95 \%$ confidence interval (shaded area) for a range of angular velocities $(0.1$ to 0.5 radians/s). Note that all of the estimated passive torque-angle responses fell within the shaded area.

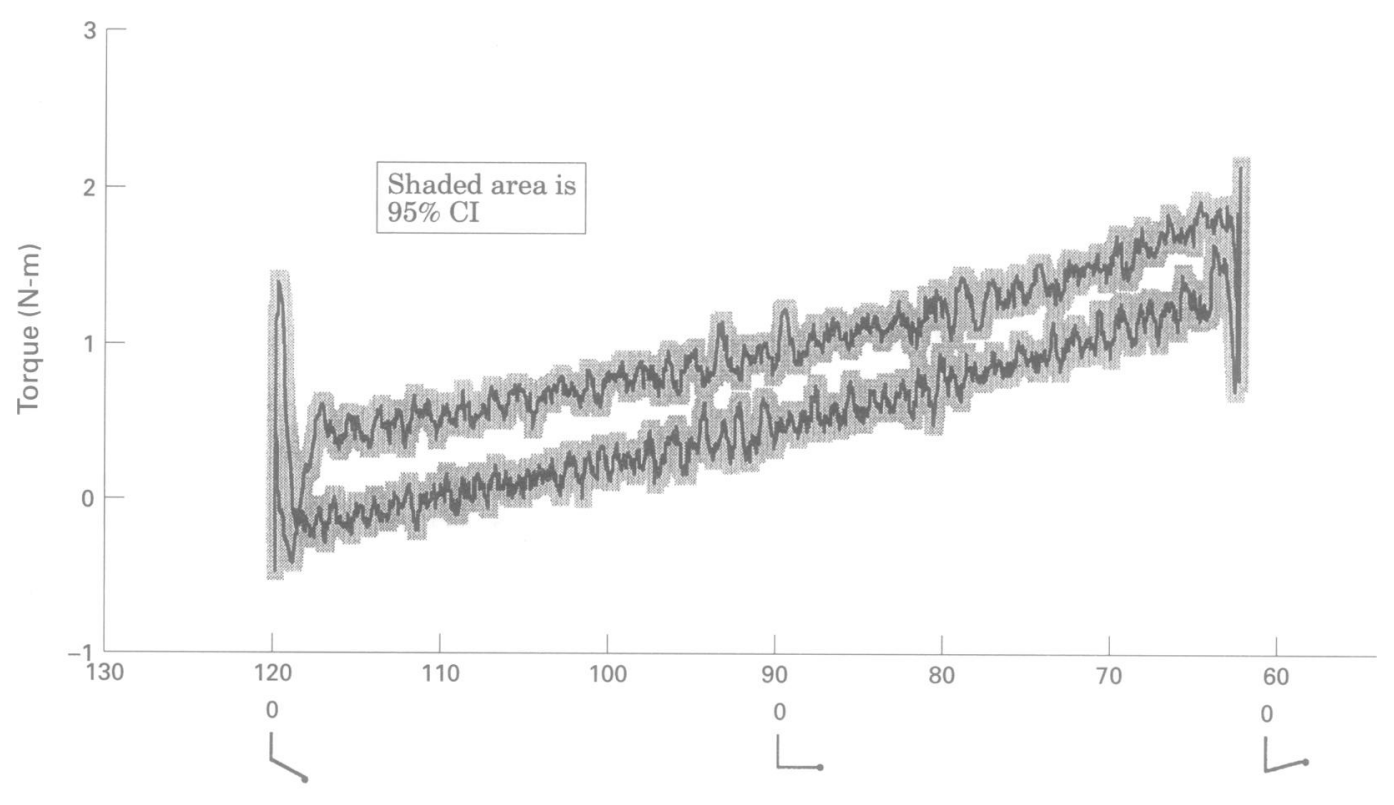




\section{Discussion}

This study presents three findings. The first, which is perhaps predictable, is that passive stiffness at the ankle is higher than at the elbow in all normal and spastic hemiparetic patients tested. The second is that ankle joints in spastic limbs showed higher stiffnesses than contralateral joints in the same patients. By contrast, no differences in passive stiffness were recorded between affected and contralateral elbow joints of hemiparetic patients. The third is that there was no significant effect of stretch velocity on the elbow passive torqueangle relations. In the following discussion, we relate these differences in torque-angle responses to known or predictable differences in muscle and joint properties at the ankle and elbow joints.

\section{JOINT DEPENDENT PASSIVE STIFFNESS IN NORMAL SUBJECTS}

Passive stiffness was found to be significantly higher at the ankle than at the elbow. This can be explained largely by the higher relative amount of intramuscular connective tissue in ankle muscles. ${ }^{11}$ Specifically, the total quantity of interstitial connective tissue (including epimysium, perimysium, ${ }^{12-14}$ and endomysium $^{15}$ is significantly higher in slow tonic muscles (such as soleus and parts of gastrocnemius muscles) than in fast muscle (such as the rectus femoris muscle). Because ankle plantar flexors especially have a high percentage of slow twitch muscle fibres, compared with elbow muscles, this could explain the differences in passive stiffness between the two joints.

Another factor contributing to the greater passive stiffness at the ankle could be the differences in cross sectional area of the ankle plantar flexors compared with either the elbow flexor or extensor muscles. ${ }^{11}$ Estimates of cross sectional area of muscles acting at the ankle ${ }^{16}{ }^{17}$ and those acting at the elbow ${ }^{18}$ showed significantly larger areas at the ankle. The cross sectional area of ankle plantar flexors is roughly 8.5 times that of the elbow flexors and 9.5 times that of the elbow extensors. Even the total area of ankle dorsiflexors is roughly twice that of either elbow flexors or extensors. The greater cross sectional area of the ankle muscles is presumably associated with greater absolute amounts of intramuscular connective tissue, and may well be an added mechanism for higher stiffnesses in these ankle muscles. ${ }^{11}$ Our own findings support this possibility, in that those with more muscle mass showed significant increases in passive stiffness about the joint (fig 4).

\section{ABSENCE OF VELOCITY EFFECTS ON PASSIVE TORQUE-ANGLE RESPONSES}

As described in the introduction, torque-angle responses recorded without concurrent voluntary or reflex muscle activation were considered to be passive. Under these conditions, no significant differences in torque-angle responses were seen at the elbow over the specified range of ramp velocities. The finding that passive torque-angle responses were invariant with increasing velocity was consistent for both the normal and hemiparetic test populations. It must be noted, however, that the range of ramp velocities differed between normal subjects and patients with hemiparetic stroke. In normal subjects, the full range of available ramp velocities was applied as even the fastest ramp velocity failed to elicit stretch reflex responses in the relaxed normal subject. In the case of patients with hemiparetic stroke, a reduced range of velocities was used to avoid activation of the stretch reflex. This varied from patient to patient depending on the patient's level of spasticity.

Peak torques were not significantly greater for a fivefold increase in velocity, there was no increase in slope of the torque-angle relation and no change in hysteresis loop area. Therefore, the system response cannot be described by a simple viscoelastic model, which would be expected to show increasing vertical separation of the extension and flexion components of the torque angle responses with increasing velocity. Because our torqueangle relations were recorded under passive conditions, it would be fair to assume that intramuscular connective tissue is primarily responsible for the observed hysteresis loop. Such strain related changes are well described in a variety of connective tissues, including, for example, the tendon and cruciate ligament, ${ }^{19}$ the cervix, ${ }^{20}$ and in passive cardiac muscle. ${ }^{21}$

Our finding of velocity insensitivity is therefore consistent with Fung ${ }^{22}$ who states that for connective tissues, which are the presumptive source of our observed torque angle relations, "the hysteresis loop is almost independent of the strain rate within several decades of the rate variation". Strain rate is the rate of change of length over the initial length which is equivalent to the velocity of stretch normalised by muscle resting length. In the case where the hysteresis loop is insensitive to velocity, Fung further states that the hysteresis is due to internal friction, although the origins of this friction are not fully identified.

In the present study, the torque differences recorded in stretch and release are thus likely to be a result of strain induced changes in the internal organisation of the tissue, such as the collagen and elastic matrix of the muscle connective tissue. ${ }^{23}$

\section{ALTERATIONS OF PASSIVE STIFFNESS IN SPASTIC PATIENTS}

We found significant increases in passive stiffness at the ankle on the impaired side of our hemiparetic stroke population, but not at the impaired elbow joint. A possible explanation for the discrepancy between the two joints may again be related to differences in immobilisation response for the different muscle fibre types found in the various muscles acting at the two joints. For example, slow twitch postural muscles at the ankle joint in rats, especially the soleus muscle, have been found to be very sensitive to immobilisation by several investigators. ${ }^{24-26}$ Their studies showed that intramuscular connective tissue increased 
more rapidly in the immobilised soleus than in the gastrocnemius muscle, which is composed primarily of fast twitch fibres. Furthermore, these increases in connective tissue were more prevalent when the muscle was kept in a shortened position. ${ }^{26}$ Similar findings were also reported by Williams and Goldspink ${ }^{27}$ who showed that passive lengthtension curves obtained after immobilisation of the rat's soleus muscle in a shortened position showed significant increases in passive stiffness which were absent when the muscle was immobilised in a lengthened position.

If rat leg muscles are an appropriate model for human leg muscles, then the alterations in passive stiffness at the human ankle could be explained by the differential sensitivity of soleus and gastrocnemius muscles to immobilisation of active muscle in a shortened position. The presence of spasticity (manifested by hyperexcitable stretch reflexes) would further facilitate a shortened muscle position and promote greater pathological changes at the joint.

EARLIER STUDIES ON PASSIVE JOINT STIFFNESS IN PATHOLGOICAL STATES

Increases in passive stiffness at the ankle joint have been reported in spastic patients by several investigators ${ }^{3-7} 28$ and therefore do match our current results. Studies considering passive stiffness in pathological conditions at the elbow are much more scarce. Increases in passive stiffness at the elbow have been reported in five of 11 patients with Parkinson's disease studied by Watts et al, ${ }^{29}$ although these investigators used a different experimental protocol, limiting possible comparisons with our data.

In cases where there are significant reductions in the passive range of motion at the elbow (a common finding in tetraplegic patients), passive elbow stiffness has been found to increase significantly. ${ }^{2}$ The difference (compared with the hemiparetic population) might be related to different muscle usage patterns or to the application of more intense physical therapy to the upper extremity of our stroke population. All our hemiparetic patients had a normal range of motion compared with the contralateral side of both the elbow and ankle joints.

\section{FUNCTIONAL IMPLICATIONS OF CURRENT}

\section{RESULTS}

The clinical implications of the animal studies reported earlier are that slow muscles, such as the soleus, are perhaps more sensitive to immobilisation than mixed or fast muscles. It follows that such slow muscles should be kept at more elongated positions to reduce the rate of connective tissue growth in cases of disuse such as occur after stroke or spinal cord injury. Clinical interventions, such as frequent elongation of the calf muscles or in extreme cases casting of the ankle joint in a dorsiflexed position, should be considered to reduce the rate of pathological changes of the muscle.

Measurement of passive stiffness also has important implications for the quantification of muscle tone abnormalities such as spastic- ity, which follow injury to the CNS. Mechanical estimates of abnormal stretch reflex activation should take account of the passive stiffness component which reflects alteration in the muscle's histological make up. Passive stiffness should be subtracted from the mechanical response when quantifying stretch reflex variables such as reflex threshold and gain (stretch reflex stiffness). The finding that passive stiffness is independent of ramp stretch velocity is an important one, as this permits stiffness estimates recorded at low velocities to be used to characterise passive stiffness at higher velocities. A characterisation of stretch reflex behaviour using this approach will permit the analysis of gain $v$ threshold changes in reflex behaviour as a function of velocity (see Given $e t a^{30}$ ).

We thank Andrew Krylow and Drs David Lloyd and C J Heckman for their valuable insights and helpful suggestions during the preparation of this manuscript. This work was supported by the following grants: Whitaker Foundation Award and NIDRR Research and Training Center for Stroke Gran H133B30024 (Project 5) to JPAD and JDG, APA Grant RB19203-1 to JDG and WZR, and NIH NS 19331 to WZR.

1 Lance JW. Pathophysiology of spasticity and clinical experience with baclofen. In: Feldman RG, Young RR rience with baclofen. In: Feldman RG, Young RR, Koella WP, eds. Spasticity: disordered

2 Given JD, Dewald JPA, Rymer WZ. Comparison of passive joint impedance in spastic hemiparetic stroke and spinal cord injured subjects with normal control subjects. Soc Neurosci 1993;19:554.

3 Dietz V, Quintern J, Berger W. Electrophysiological studies of gait in spasticity and rigidity. Brain 1981;104:431-49.

4 Broberg C, Grimby G. Measurement of torque during passive and active ankle movements in patients with muscle sive and active ankle movements in patients with muscle Med 1983;9:108-17.

5 Hufschmidt A, Mauritz KH. Chronic transformation of muscle in spasticity: a peripheral contribution to

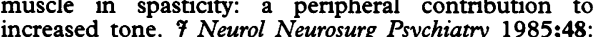
increased

6 Thilmann AF, Fellows SJ, Ross HF. Biomechanical changes at the ankle after stroke. $\mathcal{F}$ Neurol Neurosurg Psychiatry 1991;54:134-9.

7 Sinkjaer T, Toft E, Larsen K, Andreassen S, Hansen HJ. Non-reflex and reflex mediated ankle joint stiffness in multiple sclerosis patients with spasticity. Muscle Nerve 1993;16:69-76.

8 Given JD, Dewald JPA, McGuire JR, Rymer WZ. Mechanical properties of spastic muscle in hemiparetic stroke. Proceedings of the second North American Congress on biomechanics (NACOB II), Chicago, USA 1992;i:573-4.

9 Given JD, Dewald JPA, Rymer WZ. Passive ankle mechanical properties in normal and hemiparetic subjects. Second World Congress of Biomechanics Abstracts, jects. Second World Congress of Bim, Holland, 1994;1:228.

10 Ashworth RW. Preliminary trial of carisoprodol in multiple sclerosis. Practitioner 1964;192:540-2.

11 Heerkens YF, Woittiez RD, Kiela J, et al. Mechanical properties of passive rat muscle during sinusoidal stretching. Pflugers Arch 1987;409:438-47

12 Laurent GJ, Sparrow P, Bates PC, Millward DJ. Collagen content and turnover in cardiac and skeletal muscles of the adult fowl and the changes during stretch-induced growth. Biochem f 1978;176:419-27.

13 Kovanen V, Suominen H, Heikkinen E. Collagen of slow twitch and fast twitch muscle fibres in different types of rat skeletal muscle. Eur $₹ A$ Apl Physiol 1984;52:235-42.

14 Kovanen $\mathrm{V}$, Suominen $\mathrm{H}$ Peltonen $\mathrm{L}$. Effects of a life-long physical training on collagen in slow and fast skeletal muscle in rats. Cell Tissue Res 1987;248:247-55.

15 Kovanen V, Suominen H, Heikkinen E. Mechanical properties of fast and slow skeletal muscle with special reference to collagen and endurance training. $\exists$ Biomech ence to collagen

16 Wickiewicz TL, Roy RR, Powell PL, Edgerton VR Muscle architecture of the human lower limb. Clin Orthop Rel Res 1983;179:275-83

17 Friederich JA, Brand RA. Muscle fiber architecture in the human lower limb. f Biomech 1990;23:91-5.

18 An KN, Kwak BM, Chao EY, Morrey BF. Determination of muscle and joint forces: a new technique to solve the indeterminate problem. $\mathcal{F}$ Biomech Eng 1984;106:364-7.

19 Viidik A. Functional properties of collagenous tissues. International Review of Connective Tissue Research. 1973; 6:127-215.

20 Harkness MLR, Harkness RD. Changes in the physical properties of the uterine cervix of the rat during pregnancy. F Physiol 1959;148:524-47. 
21 Fung YC. Stress-strain-history relations of soft tissues in simple elongation. In: Fung YC, Perrone N, Anliker M, eds. Biomechanics: Its foundations and objectives. Englewood Cliffs, NJ : Prentice-Hall, 1972.

22 Fung YC. Biomechanics. Mechanical properties of living tissue. 2nd ed. New York: Springer-Verlag, 1993.

23 Cowan PM, North ACT, Randall JT. X-ray diffraction studies of collagen fibers. Symp Soc Exp Biol 1955;9: studies

24 Williams PE, Goldspink G. Connective tissue changes in immobilized muscle. $\mathcal{F}$ Anat 1984;138:343-50.

25 Jozsa L, Thoring J, Jarvinen M, Kannus P, Lehto M, Kvist $M$. Quantitative alterations in intramuscular connective tissue following immobilization: an experimental study in the rat calf muscles. Exp Mol Pathol 1988;49:267-78.

26 Jozsa L, Kannus P, Thoring J, Reffy A, Jarvinen M, Kvist
$M$. The effect of tenotomy and immobilization on intramuscular connective tissue. I Bone foint Surg 1990;72: 293-7.

27 Williams PE, Goldspink G. Changes in sarcomere length and physiological properties in immobilized muscle. $f$ Anat 1978;127:459-68.

28 Tardieu C, Lespargot A, Tabary C, Bret MD. Toewalking in children with cerebral palsy: contributions of contracture and excessive contraction of triceps surae muscle. Physical Therapy 1989;69:656-62.

29 Watts RL, Wiegner AW, Young RR. Elastic properties of muscles measured at the elbow in man: II. Patients with Parkinsonian rigidity. $f$ Neurol Neurosurg Psychiatry 1986;49:1177-81.

30 Given JD, Dewald JPA, Rymer WZ. Dependence of stretch reflex response on stretch velocity in spastic hemiparesis. Soc Neurosci 1994;20:339.
Samuel Jones Gee (1839-1911) and stereotypic
movements caused by apomorphia

Hughlings Jackson provides an interesting and possibly the first account of what we would now regard as a dopamine driven stereotypy. He refers, ${ }^{1}$ without the full reference, to Lauder Brunton's work on:

"the representation of the systemic sensori-motor processes concerned in vomiting in centres so high as the corpus striatum and optic thalamus."

"... when any irritation exists in the right cerebral hemisphere it will occasion vomiting more readily than irritation in the left cerebral hemisphere, and according to Budge, this is actually the case" (Lauder Brunton, Practitioner, December 1874). Dr Brunton refers to an experiment by Gee on a dog (Clinical Society Transactions 1860). Gee injected a large dose of apomorphia ( 2 grains $\left.{ }^{\star}\right)$ into a dog. The dog vomited, and in two or three minutes began to course round the room in which the experiment was performed in a curiously persistent methodical manner. Brunton (op cit) says: "This effect of apomorphia points to an action of the drug on the nervous centres, and is all the more interesting when we remember that Budge placed the cerebral centre for the stomach in the right thalamus opticus."

This paper ${ }^{2}$ of Samuel Gee is dated by Legg as 1869 . Jackson uses this experiment to illuminate the peripheral differences, chiefly of the autonomic nervous system in determining representation in the highest centres; he does not comment on the stereotypy so plainly portrayed. Gee was almost certainly the first to take the drug himself, initially doubting its emetic properties. ${ }^{3}$

Samuel Jones Gee was one of the great scholarphysicians of the 19th century. His father, a businessman had marked literary tastes, frequented the British Museum, and was prone to pilgrimages to "Cromwellian holy places". Gee attended University College School. He obtained gold medals in every subject in his medical studies at University College Hospital. Legg ${ }^{3}$ deemed his medical knowledge "encyclopaedic", his learning "prodigious". He became assistant physician to St Bartholomew's Hospital in 1866 working in the skin, anatomy, and pathological anatomy departments. His lectures were "impressive, clear, well thought out", but he was inclined to archaic expression. His aphorisms became well known to students, who mimicked his mannerisms, but probably stimulated his writing Medical Lectures and Aphorisms. He was determined in his opposition to specialisation. Legg reports that often he knew more of the rare skin diseases than the expert members of the Dermatological Society. He had a reputation for his skill in children's illnesses, but resisted attempts to make him a children's specialist.

A highly principled man of few words he none the less held strong views. Classicist, philosopher, and student of literature, he had a curious distaste for "practical men"; and politicians, he abhorred. He opposed the College of Physicians giving a license to practice to women.

His writings included many chapters in standard texts: on scarlet fever, diphtheria, and pleurisy. His Gulstonian (sic) lectures were On the Heat of the body, March 1871. Gee's Auscultation and Percussion: together with other methods of physical examination of the chest, London, James Walton 1870 , ran to a sixth edition, 1908. Medical Lectures and Aphorisms, 1902 ran to a fourth edition, 1915.

Neurological papers included: the shape of the head; spastic paraplegia; motor ataxia due to injury to the back; a case of conjugate deviation of the eyes; brassfounder's palsy; head shaking; and hereditary infantile spastic paraplegia.

$\mathrm{He}$ is often remembered for his description of infantile coeliac disease (Gee-Herter or Gee-Thaysen disease), though this was clearly depicted by Aretaeus the Cappadocian in the second century AD.

Gee was appointed physician to the Prince of Wales in 1901, and retired in 1904. In 1911 he died suddenly of a coronary occlusion, confirmed at his request by postmortem examination.

$$
\begin{array}{r}
\text { J M S PEARCE } \\
304 \text { Beverley Road, } \\
\text { Analby, Hull HU10 7BG, UK }
\end{array}
$$

1 Jackson $\mathrm{JH}$. On the scientific and empirical investigation of the epilepsies. Medical Press and Circular, October 14, 1874 to December 13, 1876. Text in: Selected writings of fohn Hughlings fackson. Vol 1. James Taylor, ed. London: Staples Press 1931:p249.

2 Gee S. On the action of a new organic base, apomorphia. Clinical Society Transactions 1869:ii; 166.

3 Gee SJ. Medical lectures and aphorisms. With recollections by J Wickham Legg. 4th ed. London: Henry Frowde and Hodder and Stoughton. 1915:357-404.

$\star 2$ grains $=120 \mathrm{mg}$. 